\title{
Fuzzy Dynamic Reliability Models of Parallel Mechanical Systems Considering Strength Degradation Path Dependence and Failure Dependence
}

\author{
Peng Gao ${ }^{1}$ and Liyang Xie ${ }^{2}$ \\ ${ }^{1}$ Department of Chemical Mechanical Engineering, Liaoning Shihua University, Liaoning 113001, China \\ ${ }^{2}$ Department of Mechanical Engineering and Automation, Northeastern University, Liaoning 110004, China \\ Correspondence should be addressed to Peng Gao; gaogaopeng@163.com
}

Received 27 June 2014; Revised 18 September 2014; Accepted 5 October 2014

Academic Editor: Enrico Zio

Copyright (C) 2015 P. Gao and L. Xie. This is an open access article distributed under the Creative Commons Attribution License, which permits unrestricted use, distribution, and reproduction in any medium, provided the original work is properly cited.

Fuzzy dynamic reliability models of mechanical parallel systems with respect to stress parameters and strength parameters are developed in this paper. Strength degradation path dependence (SDPD) and failure dependence of components in the system are two main problems to be addressed in developing fuzzy dynamic reliability of mechanical systems, which are taken into account in the proposed reliability models. In addition, the SDPD sensitivity function and the failure dependence sensitivity function are defined to analyze the influences of the fuzzy characteristics of stress on the effects of SDPD and failure dependence of mechanical components on dynamic reliability. The bolted joint systems for connection between launch vehicle and satellite are chosen as illustrative examples to demonstrate the proposed model. Moreover, Monte Carlo simulations are carried out to validate the effectiveness of the proposed models. The results show that SDPD and failure dependence of components have significant impacts on fuzzy dynamic reliability of mechanical systems. Besides, the influences of the fuzzy characteristics of stress on SDPD sensitivity and that on failure dependence sensitivity are different.

\section{Introduction}

Over the last few decades, reliability models of mechanical systems have been well reported in the probability context [1-5]. The basic idea for reliability analysis of mechanical systems is to calculate the system reliability according to the component reliability in the system and structure function (SF). For mechanical components, the well-known stressstrength interference (SSI) model is the most important theory for reliability assessment, where both stress and strength are modeled as random variables. In the SSI model, the failure of mechanical components is defined as the probability that the stress is higher than strength as shown in Figure 1. The interference area between the probability density function (pdf) curve of stress and the pdf curve of strength is an indication of the possibility that the failure of the component might occur.

However, for mechanical systems in aerospace products, failures are relatively rare events. It is a costly and difficult undertaking to collect enough data to calculate the probability of failure. Besides, due to the complexity of the operational environment, it is difficult to obtain a large number of samples to precisely describe the statistical characteristics of stress caused by environmental load on the mechanical systems in aerospace products, especially in the preliminary design stage where many factors have to be determined by the judgment of engineers. In this case, it can only increase the uncertainty in reliability estimation to adopt the imprecise pdf of stress. Alternatively, fuzzy theory can be used to deal with this problem by modeling stress in an analytical form via integrating the limited available data and expert judgments.

As a matter of fact, fuzzy variables are always used to deal with the uncertainty brought by insufficient information. In this paper, we model the stress at each load application as a fuzzy variable. We assume that the uncertainty of stress comes from the insufficient information about the stress. When the amount of available information increases, which is reflected via the enhancement of membership degree, the uncertainty 


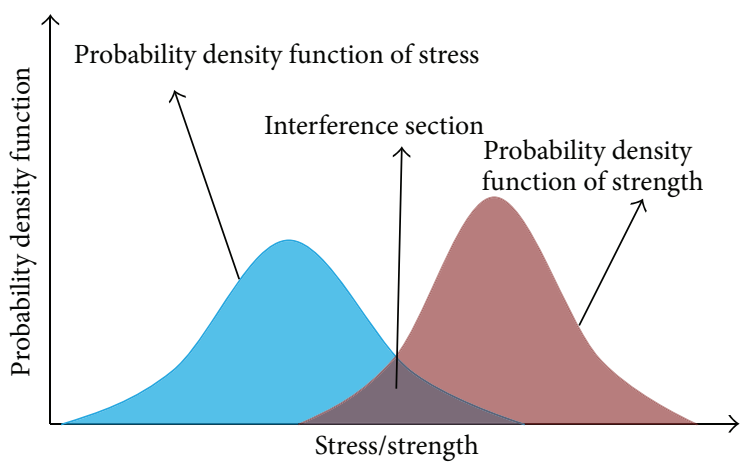

FIGURE 1: Schematic SSI model.
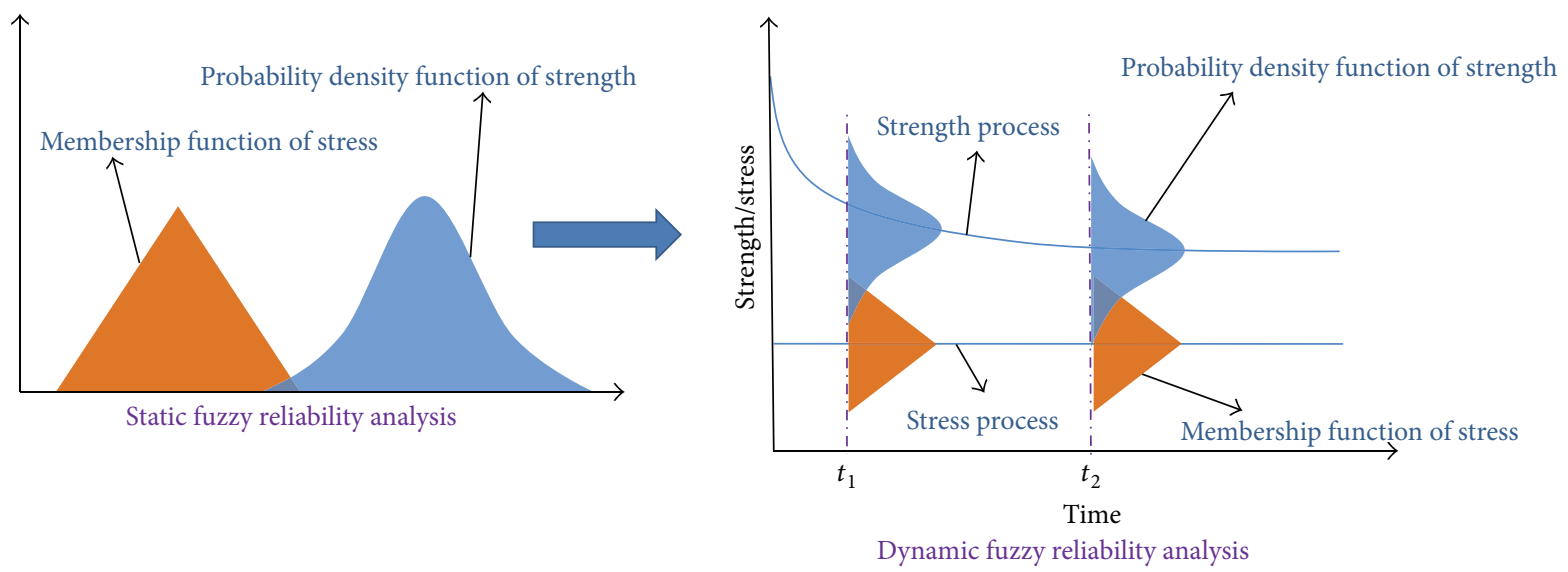

FIGURE 2: Schematic stress process and strength process.

of stress decreases and the stress tends to be a deterministic number.

Since Zadeh introduced the fuzzy theory in 1965 [6], great efforts have been made to develop fuzzy reliability models. Cai et al. classified the fuzzy reliability models into three basic classes: the robust reliability models, the posbist reliability models, and the posfust reliability models [7, 8]. Besides, Cai et al. proposed fuzzy reliability models of various typical systems based on the possibility assumption and the fuzzy state assumption [9]. Wu discussed the relationship between the fuzzy reliability of a system and the fuzzy reliability of its components via the proposed fuzzy reliability models [10]. Utkin proposed the method for fuzzy reliability analysis and fuzzy reliability optimization [11]. Huang et al. presented an approach for reliability estimation by using the Bayesian method based on fuzzy lifetime data [12]. Bing et al. extended the conventional SSI model to the fuzzy stress-random strength interference model to analyze the fuzzy reliability of mechanical components [13]. These fuzzy reliability models establish the basis and framework for reliability assessment in the possibility context. However, for mechanical systems, it is difficult to calculate reliability by directly employing these models because of the problems as follows.

(1) Strength degradation exists commonly for mechanical components in practical engineering. The interaction between the stress and the strength is a dynamic process. Most of current fuzzy reliability models of mechanical components and mechanical systems are static, which are only suitable for the reliability calculation at a specific moment as shown in Figure 2. As mentioned in the authors' former research in [14], when considering the strength degradation process of mechanical components, the strength degradation path dependence (SDPD) is a main problem to be addressed in developing dynamic reliability of mechanical components, which also exists in establishing fuzzy reliability models. As a matter of fact, fuzzy dynamic reliability models of parallel mechanical systems considering SDPD is seldom reported. Besides, the fuzzy characteristics of stress have great influences on SDPD, which is also seldom reported in current literatures. In this paper, impacts of fuzzy characteristics of stress on the effects of SDPD on reliability will be analyzed via the proposed fuzzy dynamic reliability models.

(2) Due to the unique operating mode of mechanical product, failure dependence exists commonly in mechanical systems and the components, under the stress with a common source of load, in the system are statistically dependent on each other. Fuzzy dynamic reliability models of parallel mechanical systems with 
respect to stress and material parameters, which consider failure dependence between different components in a system, are seldom reported. Correspondingly, the influences of fuzzy characteristics of stress on failure dependence need further investigation. In this paper, the sensitivity analysis of dynamic system reliability with respect to fuzzy parameters of stress will be performed in order to present how the fuzzy characteristics of stress affect the failure dependence behavior of components in mechanical systems.

The remainder of this paper is organized as follows. In Section 2, fuzzy dynamic reliability models of parallel mechanical systems are developed, which take SDPD and failure dependence into consideration. In Section 3, Monte Carlo simulations are carried out to verify the reliability models. Furthermore, the influences of fuzzy characteristics of stress on both the effects of SDPD and the effects of failure dependence on reliability are analyzed via the numerical examples.

\section{Fuzzy Dynamic Reliability Models of Parallel Mechanical Systems}

Denote the pdf of a random variable $x$ by $f(x)$ and the membership function (MF) of $x$ belonging to a fuzzy set $\widetilde{X}$ by $\mu_{\widetilde{X}}(x)$. Then, the fuzzy probability that $x$ belongs to $\widetilde{X}$ can be calculated as [6]

$$
P(x)=\int_{-\infty}^{\infty} \mu_{\widetilde{X}}(x) f(x) d x
$$

Meanwhile, the fuzzy probability in (1) can also be calculated by employing the $\alpha$-cut of fuzzy set as follows:

$$
P(x)=\int_{0}^{1} \int_{\widetilde{X}_{\alpha}^{L}}^{\widetilde{X}_{\alpha}^{U}} f(x) d x d \alpha .
$$

In fact, the expression of fuzzy probability in (2) provides the basis for reliability evaluation with multiple format of variables, such as the random variables and fuzzy variables, involved in the calculation. As mentioned above, in some cases, it is difficult to obtain the accurate pdf of stress on components in mechanical systems because of insufficient load history samples, while most pdf of material parameters can be acquired from handbooks for mechanical design or experiment. Therefore, in this paper, the stress is modeled as a fuzzy variable and strength is modeled as a random variable. As a matter of fact, the triangular membership function (TMF) is always adopted to express the fuzzy characteristics of stress and the parameters in TMF of stress $s$ can be evaluated by using the method in [13]. The mathematical expression of TMF of $s$ can be given by

$$
\mu_{s}(s)= \begin{cases}\frac{s-a}{b-a}, & a \leq s<b, \\ 1, & s=b, \\ \frac{s-c}{b-c}, & b \leq s<c, \\ 0, & \text { otherwise. }\end{cases}
$$

The TMF can also be expressed as a triplet $(a, b, c)$ for descriptive convenience. In addition, appoint the MF to be equal to a specific value of $\alpha$. Then, the elements with degree of membership larger than $\alpha$ constitute an $\alpha$-cut. Through the $\alpha$-cut, the computation under fuzzy uncertainty can be transformed into the computation under the interval uncertainty. Correspondingly, the calculation with respect to fuzzy numbers can be reduced to the calculation with respect to interval numbers. To consider the weight of elements on a specified interval and for the purpose of computational convenience in case of both random variables and interval variables existing, a certain distribution is sometimes assumed for the elements on the interval, such as the uniform distribution $[15,16]$ or the linear distribution [17]. As pointed out by Nguyen et al. when the distribution is unknown on a given interval, the maximum entropy approach is always used to determine an appropriate distribution for the elements on the interval. In the case where the uniform distribution is adopted, the entropy reaches its maximum value [18]. In this paper, we assign uniform distribution to the elements in an $\alpha$-cut and denote the fuzzy stress at the $i$ th load application by $\left(a_{i}, b_{i}, c_{i}\right)$. Then, the distribution function of the $\alpha$-cut can be given by

$$
\begin{aligned}
& F_{i \alpha}\left(s_{i}\right) \\
& \quad=\left\{\begin{array}{lr}
0, & s_{i}<a_{i}+\left(b_{i}-a_{i}\right) \alpha, \\
\frac{s_{i}-a_{i}-\left(b_{i}-a_{i}\right) \alpha}{(1-\alpha)\left(c_{i}-a_{i}\right)}, & a_{i}+\left(b_{i}-a_{i}\right) \alpha \leq s_{i} \\
& <c_{i}-\left(c_{i}-b_{i}\right) \alpha, \\
1, & s_{i} \geq c_{i}-\left(c_{i}-b_{i}\right) \alpha .
\end{array}\right.
\end{aligned}
$$

According to the reliability theory of systems, a parallel system fails to operate when all the components in the system fail to work. In this paper, we assume that there are $k$ identical components with the same structure and with the strength pdf of $f_{r}(r)$ in a parallel system. When the components in a parallel system are subjected to the same load, the stress of $s$ with the pdf of $f_{s}(s)$ on each component is mutually identical. Then, the probability that the parallel system does not fail to work is equal to the probability that the maximum strength is higher than $s$, which can be expressed by using the order statistics theory as follows:

$$
P\left(r_{\max }>s\right)=\int_{s}^{\infty} k\left[\int_{-\infty}^{r} f_{r}(r) d r\right]^{k-1} f_{r}(r) d r
$$

Therefore, the reliability of the parallel system can be given by

$$
R_{\text {parallel }}=\int_{-\infty}^{\infty} f_{s}(s)\left\{\int_{s}^{\infty} k\left[\int_{-\infty}^{r} f_{r}(r) d r\right]^{k-1} f_{r}(r) d r\right\} d s .
$$


Equation (6) is similar to the SSI model in mathematical expression. Hence, the equivalent strength of the parallel system can be expressed as

$$
f_{\text {parallel }}(r)=k\left[\int_{-\infty}^{r} f_{r}(r) d r\right]^{k-1} f_{r}(r) .
$$

In the failure mode of fatigue, strength could degrade under the application of random load which causes damage to the components in the system. The remaining strength is the function of the load application times and the magnitude of stress, which is generally expressed as follows [14]:

$$
r(n)=r_{0}[1-D(n)]^{\gamma}
$$

where $r_{0}$ and $\gamma$ are initial strength and material parameter, respectively. $D(n)$ is the cumulative damage induced by the applied load. In addition, the $S-N$ Curve, which is usually adopted to compute the lifetime of mechanical components under the stress with a specific magnitude, can be written in an analytical format as follows:

$$
s^{m} N=C,
$$

where $m$ and $C$ are material parameters. When considering the effects of SDPD, the equivalent remaining strength under the stress at the level of $\alpha$ can be obtained according to the rule of damage accumulation and the rule of damage equivalence as follows [14]:

$$
\begin{aligned}
& r_{\alpha}(n)=r_{0}\left\{1-\left\{\sum_{i=1}^{n}\{\right.\right.\left(\left[c_{i}-\left(c_{i}-b_{i}\right) \alpha\right]^{m+1}\right. \\
&\left.-\left[a_{i}+\left(b_{i}-a_{i}\right) \alpha\right]^{m+1}\right) \\
&\left.\left.\left.\times\left((m+1)(1-\alpha)\left(c_{i}-a_{i}\right) C\right)^{-1}\right\}\right\}^{\gamma}\right\} .
\end{aligned}
$$

When the initial strength $r_{0}$ is deterministic, the reliability at the $i$ th load application can be given according to the distribution of the fuzzy stress on the interval of $\alpha$-cut as follows:

$$
\begin{aligned}
& R_{i} \\
& =\left\{\begin{array}{lc}
0, & r_{\alpha}(n-1)<a_{i}+\left(b_{i}-a_{i}\right) \alpha, \\
\frac{r_{\alpha}(n-1)-a_{i}-\left(b_{i}-a_{i}\right) \alpha}{(1-\alpha)\left(c_{i}-a_{i}\right)}, & a_{i}+\left(b_{i}-a_{i}\right) \alpha \leq r_{\alpha}(n-1) \\
& <c_{i}-\left(c_{i}-b_{i}\right) \alpha, \\
1, & r_{\alpha}(n-1) \geq c_{i}-\left(c_{i}-b_{i}\right) \alpha .
\end{array}\right.
\end{aligned}
$$

Therefore, the probability that the system operates normally when load applies for $n$ times can be expressed as

$$
R_{\alpha}(n)=\prod_{i=1}^{n} R_{i}
$$

When considering the distribution of the equivalent strength of the system characterized by the pdf shown in (7), the fuzzy reliability of the parallel system at the level of $\alpha$ can be obtained according to the total probability formula for continuous variables as follows:

$$
R_{\alpha}(n)=\int_{-\infty}^{\infty} k\left[\int_{-\infty}^{r_{0}} f_{r_{0}}\left(r_{0}\right) d r_{0}\right]^{k-1} f_{r_{0}}\left(r_{0}\right) \prod_{i=1}^{n} R_{i} d r_{0}
$$

According to the decomposition theorem, the fuzzy dynamic reliability of the parallel system can be given by

$$
R(n)=\int_{0}^{1} \int_{-\infty}^{\infty} k\left[\int_{-\infty}^{r_{0}} f_{r_{0}}\left(r_{0}\right) d r_{0}\right]^{k-1} f_{r_{0}}\left(r_{0}\right) \prod_{i=1}^{n} R_{i} d r_{0} d \alpha
$$

Hence, the failure rate of the parallel system can be expressed as follows:

$\lambda(n)$

$$
\begin{aligned}
= & \left(\int_{0}^{1} \int_{-\infty}^{\infty} k\left[\int_{-\infty}^{r_{0}} f_{r_{0}}\left(r_{0}\right) d r_{0}\right]^{k-1} f_{r_{0}}\left(r_{0}\right) \prod_{i=1}^{n} R_{i} d r_{0} d \alpha\right. \\
& \left.-\int_{0}^{1} \int_{-\infty}^{\infty} k\left[\int_{-\infty}^{r_{0}} f_{r_{0}}\left(r_{0}\right) d r_{0}\right]^{k-1} f_{r_{0}}\left(r_{0}\right) \prod_{i=1}^{n+1} R_{i} d r_{0} d \alpha\right) \\
& \times\left(\int_{0}^{1} \int_{-\infty}^{\infty} k\left[\int_{-\infty}^{r_{0}} f_{r_{0}}\left(r_{0}\right) d r_{0}\right]^{k-1} f_{r_{0}}\left(r_{0}\right) \prod_{i=1}^{n} R_{i} d r_{0} d \alpha\right)^{-1} .
\end{aligned}
$$

It should be noted that (14) is derived with the effects of SDPD taken into consideration. For computational convenience, conventional dynamic reliability models always model the strength degradation process as a stochastic process with the distribution of strength at each load application determined by the specified stochastic process and calculate the reliability based on the strength at each load application. As pointed by the authors' former study, it could result in large computational error due to neglecting the effects of SDPD [14]. When the fuzzy system reliability is calculated according to the distribution of component strength at each load application, which can be obtained from the distribution of initial strength and (10), the fuzzy reliability can be calculated as follows:

$$
R_{1}(n)=\int_{0}^{1} \prod_{i=1}^{n} \int_{-\infty}^{\infty} f_{i \alpha}\left(s_{i}\right)\left[1-\left(\int_{-\infty}^{s} f_{r_{i}}\left(r_{i}\right) d r_{i}\right)^{k}\right] d s d \alpha,
$$

where $f_{i \alpha}\left(s_{i}\right)$, which can be obtained via derivation of $F_{i \alpha}\left(s_{i}\right)$ in (4) with respect to $s_{i}$, is the pdf of stress caused by the $i$ th load application and $f_{r_{i}}\left(r_{i}\right)$ is the pdf of strength at the $i$ th load application.

As a matter of fact, the fuzzy characteristics of stress are mainly determined by the parameters of $a, b$, and $c . b$ is the center parameter with the largest membership degree. When more information is available, which means more investment 
TABLE 1: Stress parameters and material parameters of explosive bolts.

\begin{tabular}{lccccccc}
\hline$\mu\left(r_{0}\right)[\mathrm{MPa}]$ & $\sigma\left(r_{0}\right)[\mathrm{MPa}]$ & $a[\mathrm{MPa}]$ & $b[\mathrm{MPa}]$ & $c[\mathrm{MPa}]$ & $m$ & $\gamma$ & $C\left[\mathrm{MPa}{ }^{2}\right]$ \\
\hline 400 & 5 & 250 & 300 & 350 & 2 & 1 & $10^{8}$ \\
\hline
\end{tabular}

in experiments, the parameters of $a, c$ will be closer to $b$ and the fuzzy number tends to be a deterministic number. In order to analyze the influences of the parameters of $a$ and $c$ on the effects of SDPD on reliability, the SDPD sensitivity functions with respect to $a$ and $c$ are defined as follows:

$I_{\mathrm{SDPD} a}(n)$

$$
\begin{aligned}
= & \frac{\partial\left[R(n)-R_{1}(n)\right]}{\partial a} \\
= & \frac{\partial\left\{\int_{0}^{1} \int_{-\infty}^{\infty} k\left[\int_{-\infty}^{r_{0}} f_{r_{0}}\left(r_{0}\right) d r_{0}\right]^{k-1} f_{r_{0}}\left(r_{0}\right) \prod_{i=1}^{n} R_{i} d r_{0} d \alpha\right\}}{\partial a} \\
& -\frac{\partial\left\{\int_{0}^{1} \prod_{i=1}^{n} \int_{-\infty}^{\infty} f_{i \alpha}\left(s_{i}\right)\left[1-\left(\int_{-\infty}^{s} f_{r_{i}}\left(r_{i}\right) d r_{i}\right)^{k}\right] d s d \alpha\right\}}{\partial a},
\end{aligned}
$$

$$
\begin{aligned}
& I_{\mathrm{SDPD} c}(n) \\
&=\frac{\partial\left[R(n)-R_{1}(n)\right]}{\partial c} \\
&=\frac{\partial\left\{\int_{0}^{1} \int_{-\infty}^{\infty} k\left[\int_{-\infty}^{r_{0}} f_{r_{0}}\left(r_{0}\right) d r_{0}\right]^{k-1} f_{r_{0}}\left(r_{0}\right) \prod_{i=1}^{n} R_{i} d r_{0} d \alpha\right\}}{\partial c} \\
&-\frac{\partial\left\{\int_{0}^{1} \prod_{i=1}^{n} \int_{-\infty}^{\infty} f_{i \alpha}\left(s_{i}\right)\left[1-\left(\int_{-\infty}^{s} f_{r_{i}}\left(r_{i}\right) d r_{i}\right)^{k}\right] d s d \alpha\right\}}{\partial c} .
\end{aligned}
$$

In addition, when the components in the system are mutually independent, the fuzzy reliability can be computed as

$$
R_{2}(n)=1-\left(1-\int_{0}^{1} \int_{-\infty}^{\infty} f_{r_{0}}\left(r_{0}\right) \prod_{i=1}^{n} R_{i} d r_{0} d \alpha\right)^{k}
$$

In order to evaluate the impacts of the parameters of $a$, $c$ on failure dependence, we define the failure dependence sensitivity functions with respect to $a$ and $c$ as follows:

$$
\begin{aligned}
I_{\mathrm{FD} a}(n) & \\
= & \frac{\partial\left[R(n)-R_{2}(n)\right]}{\partial a} \\
= & \frac{\partial\left\{\int_{-\infty}^{\infty} k\left[\int_{-\infty}^{r_{0}} f_{r_{0}}\left(r_{0}\right) d r_{0}\right]^{k-1} f_{r_{0}}\left(r_{0}\right) \prod_{i=1}^{n} R_{i} d r_{0}\right\}}{\partial a} \\
& -\left(k\left(1-\int_{-\infty}^{\infty} f_{r_{0}}\left(r_{0}\right) \prod_{i=1}^{n} R_{i} d r_{0}\right)^{k-1}\right. \\
& \left.\quad \times \partial\left\{\int_{0}^{1} \int_{-\infty}^{\infty} f_{r_{0}}\left(r_{0}\right) \prod_{i=1}^{n} R_{i} d r_{0} d \alpha\right\}\right) \times(\partial a)^{-1},
\end{aligned}
$$

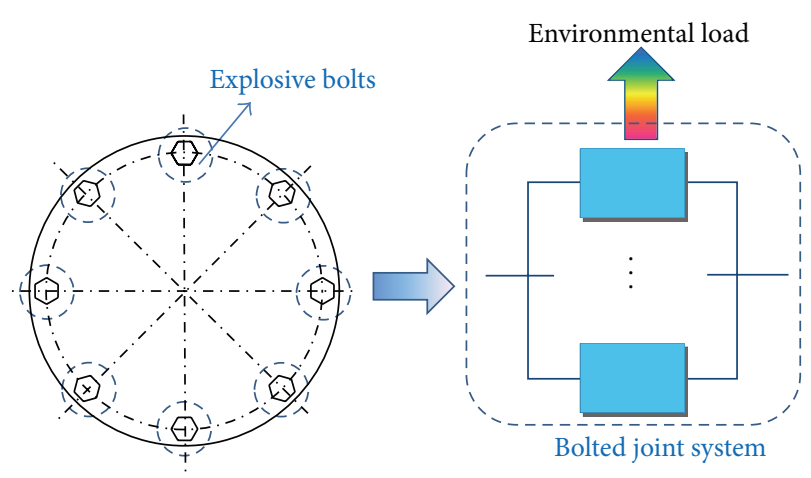

FIgURE 3: The bolted joint system.

$$
\begin{aligned}
I_{\mathrm{FD} c}(n) & \\
= & \frac{\partial\left[R(n)-R_{2}(n)\right]}{\partial c} \\
= & \frac{\partial\left\{\int_{-\infty}^{\infty} k\left[\int_{-\infty}^{r_{0}} f_{r_{0}}\left(r_{0}\right) d r_{0}\right]^{k-1} f_{r_{0}}\left(r_{0}\right) \prod_{i=1}^{n} R_{i} d r_{0}\right\}}{\partial c} \\
& -\left(k\left(1-\int_{-\infty}^{\infty} f_{r_{0}}\left(r_{0}\right) \prod_{i=1}^{n} R_{i} d r_{0}\right)^{k-1}\right. \\
& \left.\times \partial\left\{\int_{0}^{1} \int_{-\infty}^{\infty} f_{r_{0}}\left(r_{0}\right) \prod_{i=1}^{n} R_{i} d r_{0} d \alpha\right\}\right) \times(\partial c)^{-1} .
\end{aligned}
$$

\section{Illustrative Examples}

The explosive bolts are comprehensively used for connection and separation in aerospace products. For example, the bolted joints for connection between launch vehicle and satellite are shown in Figure 3. The redundancy design of the explosive bolt system largely improves the reliability of the success launch of satellites. In this section, the bolted joint system is chosen as an illustrative example to analyze the influence of the fuzzy characteristics of stress on SDPD and failure dependence of parallel mechanical systems. In the bolted joint system, the explosive bolts are mutually identical with the material parameters [14] and stress parameters listed in Table 1 .

In this section, three cases will be analyzed in order to investigate the following problems:

(1) verify the proposed model via Monte Carlo simulation;

(2) analyze the influences of the parameters of $a, c$ on the effects of SDPD on reliability; 


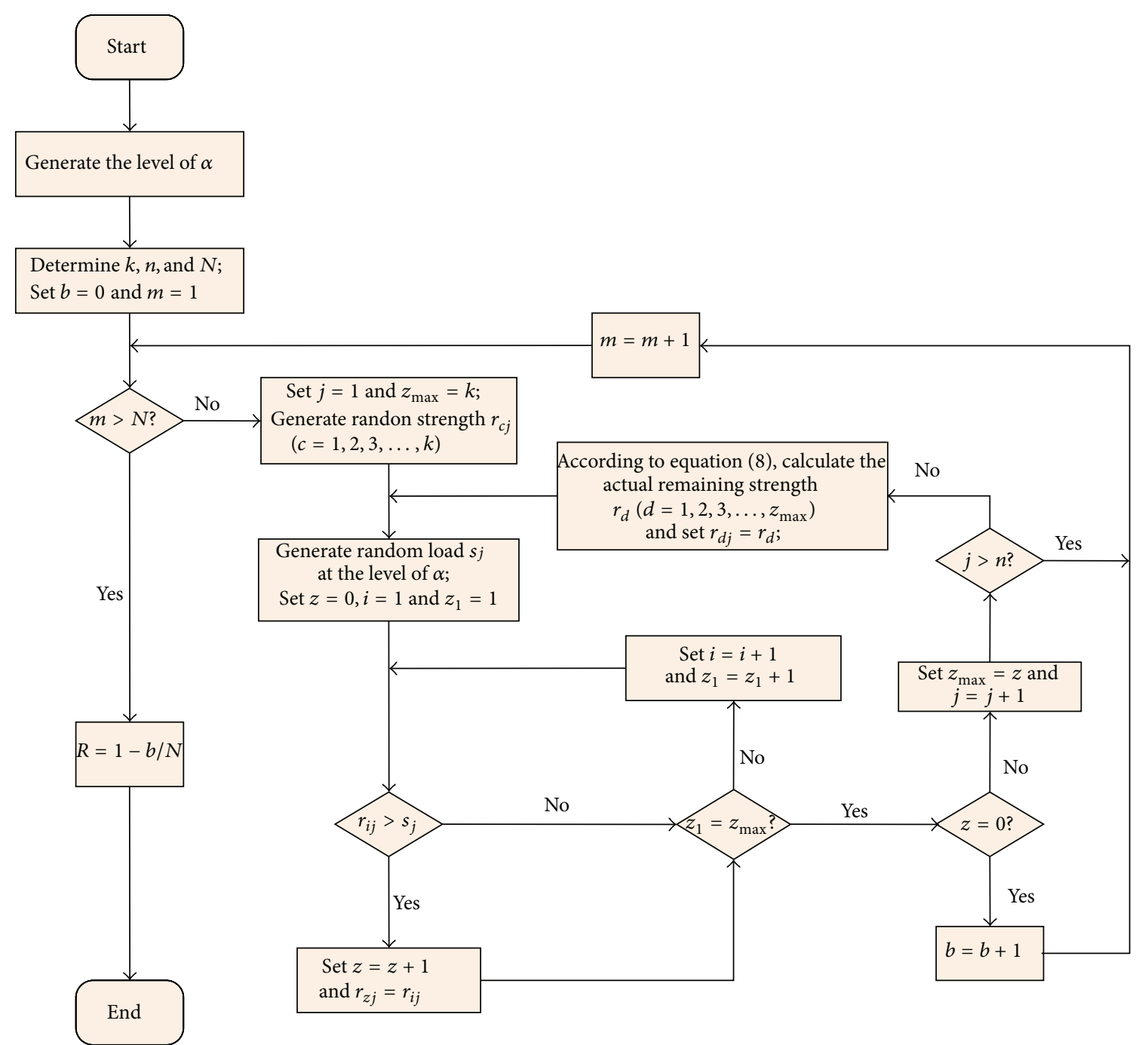

Figure 4: Flowchart of the Monte Carlo simulation for parallel subsystem.

(3) investigate the influences of the parameters of $a, c$ on the failure dependence.

Case 1. Consider a system with three dependent components. The flowchart for the Monte Carlo simulation is shown in Figure 4 . In the flowchart, $k$ represents the number of components in the system. $N$ is the total number of simulation trials. $n$ is the number of load application. $b$ is the total number of failure of the system in the $N$ trials. It should be noted that in the Monte Carlo simulation, the strength degradation process is only dependent on the randomly generated stress and (8) for remaining strength calculation under deterministic stress. Therefore, the simulation is essentially identical with physical experiment and does not rely on any analytical dynamic reliability model. The comparison between the result of the Monte Carlo simulation and the result from the proposed models is shown in Figure 5.

From Figure 5, it can be learnt that the result from the proposed reliability method shows good agreement with the reliability from Monte Carlo simulations. The reliability decreases rapidly with the load application.
Case 2. Consider a system with three dependent components. The system reliability considering SDPD and the system reliability without SDPD taken into account are shown in Figure 6. In addition, the SDPD sensitivity with respect to $a$ and $c$ is shown in Figures 7 and 8, respectively.

From Figure 6, it can be seen that SDPD have great influences on fuzzy system reliability. Although it could facilitate the reliability calculation by directly using the strength distribution at each load application, reliability could be underestimated due to neglecting the effects of SDPD. Moreover, from Figures 7 and 8 , it can be learnt that the left parameter $a$ and right parameter $c$ have different impacts on the effects of SDPD on reliability. The effects of SDPD on reliability are more sensitive to variation of $c$.

For a deterministic $a$, the local maximum of the error caused by SDPD arises at middle stage and the end of the operational duration of the system. The reliability calculated considering SDPD is higher than that without SDPD taken into account. Besides, from (17) and (18), we can learn that, for the SDPD sensitivity, a positive value means that the error increases with the increase of the parameter considered 


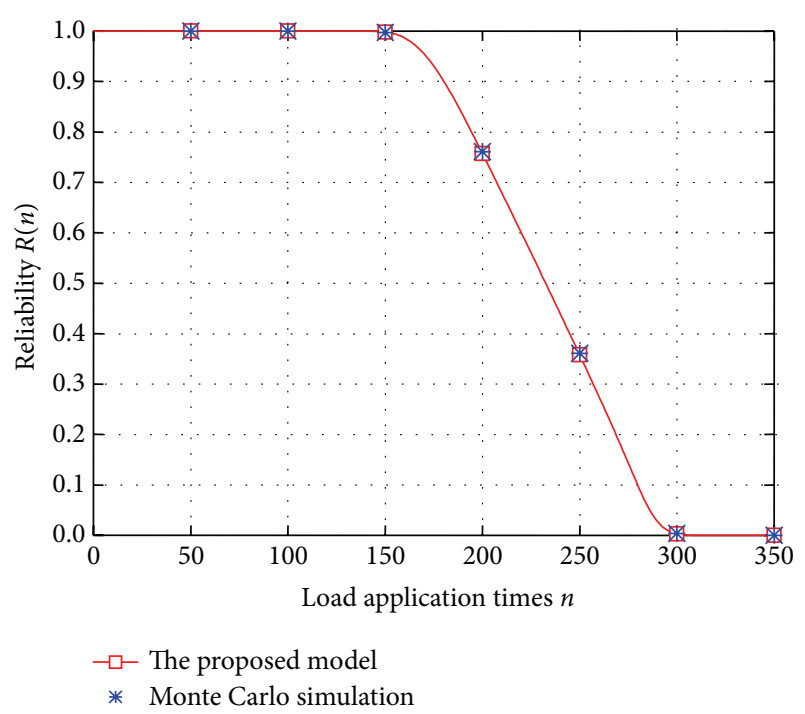

FIgURE 5: Reliability from Monte Carlo simulation and reliability from proposed models.

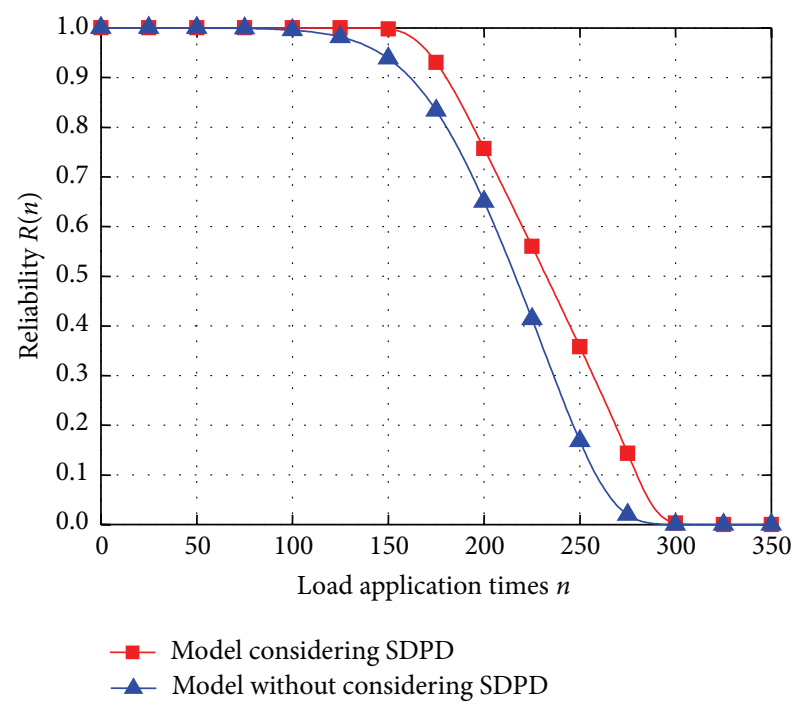

FIGURE 6: Reliability considering SDPD and reliability without SDPD taken into consideration.

( $a$ or $c$ ), while a negative value means that the error decreases with the increase of the parameter considered ( $a$ or $c$ ). Moreover, the reliability calculated considering SDPD is always larger than the reliability calculated without SDPD taken into account. Therefore, the SDPD sensitivity with a negative value means that the effects of SDPD on reliability are weakened. In mechanical design, engineers are more interested in the sensitive areas where a small variation in the parameter considered could lead to a rapid increase in the error due to neglecting SDPD. Thus, in this paper, we are concentrated on the characteristics of the positive peak value of SDPD sensitivity. From Figure 7, we can see that two evident positive peak values of the SDPD sensitivity appear in the middle stage of the system operational duration,

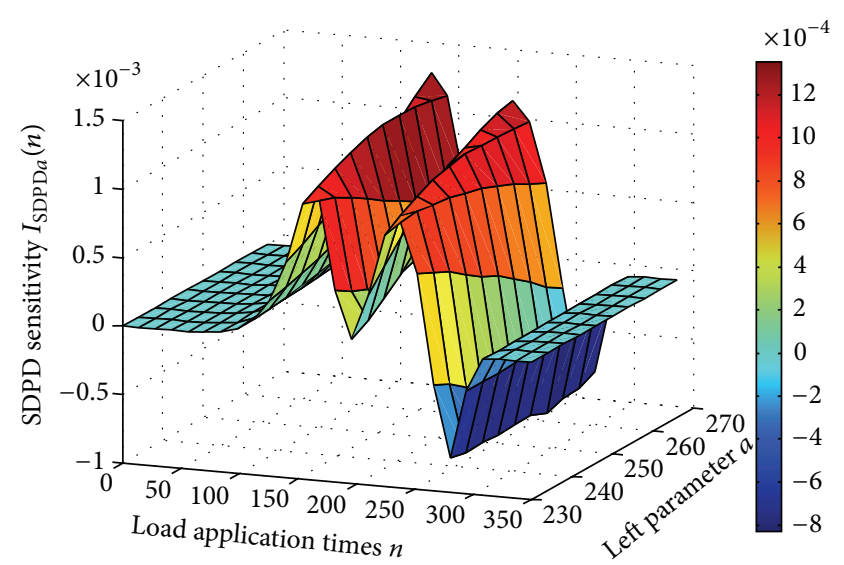

FIGURE 7: SDPD sensitivity with respect to $a$.

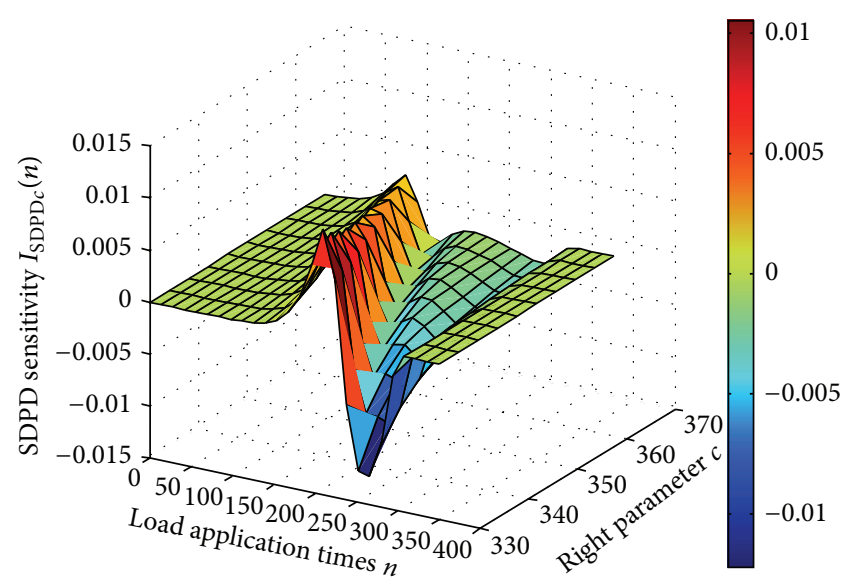

FIGURE 8: SDPD sensitivity with respect to $c$.

which represent the most sensitive time instant. Therefore, in dynamic system reliability analysis, attention should be paid to the influences of the change in $a$ on the effects of SDPD at these two sensitive "time instants" associated with their adjacent time interval. In addition, the positive peak value of the SDPD sensitivity comes earlier when $a$ increases.

For a deterministic $c$ with a low value, only one positive peak value of the SDPD sensitivity appears in the middle stage of the system operational duration. However, when $c$ is large, two positive peak values arise in the middle stage of the system operational duration with one peak value evidently larger than the other one. Therefore, we should pay attention to the variation of $c$ at the moment where the positive peak value of the SDPD sensitivity appears in the reliability estimation when considering the effects of SDPD. Similar to the case in the sensitivity analysis with respect to $a$, the maximum of the SDPD sensitivity comes earlier when $c$ increases.

Case 3. In this section, we are aimed at analyzing the influences of the failure dependence of components on system reliability and influences of fuzzy parameters of stress on failure dependence sensitivity function. The system 


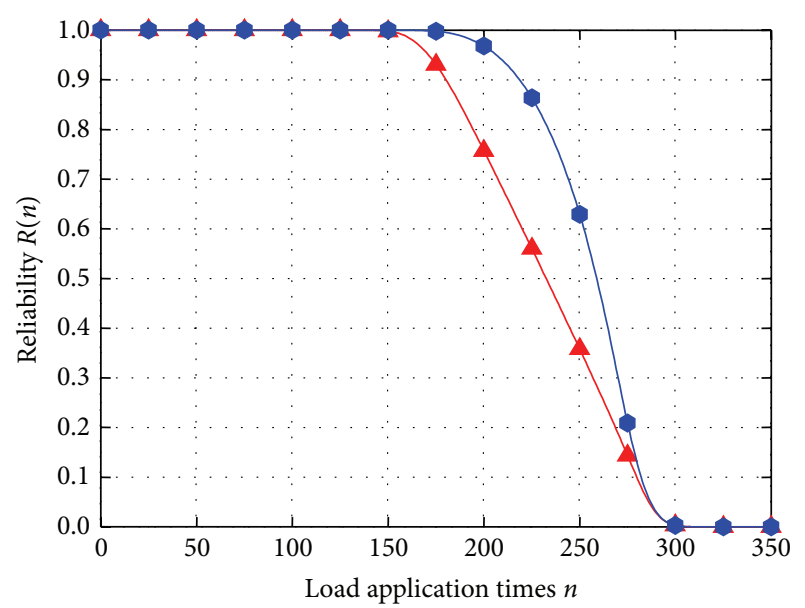

- - System with dependent components

- System with independent components

FIGURE 9: Reliability of dependent system and reliability of independent system.

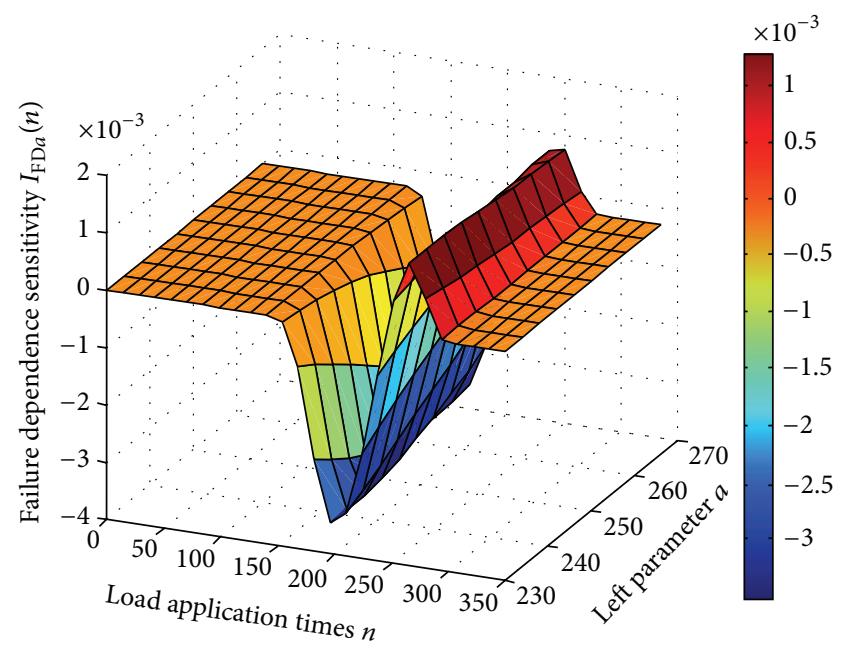

FIGURE 10: Failure dependence sensitivity with respect to $a$.

reliability considering failure dependence of components can be calculated according to (14), while the system reliability under the assumption that the components are independent of each other can be calculated according to (19). The failure dependence sensitivity function with respect to $a$ and $c$ can be obtained through (20). The reliability of a system with three dependent components and the reliability of a system with three independent components are shown in Figure 9. In addition, the failure dependence sensitivity with respect to $a$ and $c$ is shown in Figures 10 and 11, respectively.

From Figure 9, it can be seen that the failure dependence makes the parallel system less reliable. Thus, from (18) and (19), Figures 10 and 11, it can be seen that the negative peak value of the failure dependence sensitivity means that the error caused by failure dependence increases at the highest speed, while the positive peak value of the SDPD sensitivity means that the error decreases at the highest speed. Therefore,

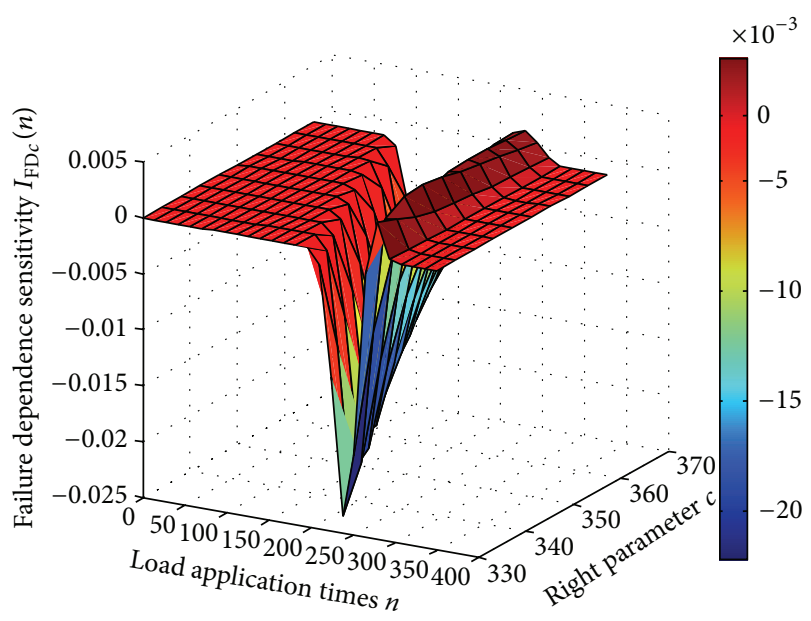

FIGURE 11: Failure dependence sensitivity with respect to $c$.

we are more concentrated on the negative peak value of the failure dependence sensitivity. A single negative peak value of the failure dependence sensitivity appears in the middle stage of the system operational duration in both the case of failure dependence sensitivity analysis with respect to $a$ and the case of failure dependence sensitivity analysis with respect to $c$. We should pay attention to the variation of $a$ and $c$ at the moment where the negative peak value of the failure dependence sensitivity appears in the reliability assessment when considering the effects of failure dependence. Besides, the negative peak value of the failure dependence sensitivity comes earlier when $a$ or $c$ increases. In general, the effects of the failure dependence of components in the parallel system are more sensitive to the change of $c$.

\section{Conclusion}

In this paper, fuzzy dynamic reliability models of mechanical parallel systems with respect to stress parameters and strength parameters are established. In the proposed reliability models, the SDPD and failure dependence of components in a mechanical parallel system are taken into account. For computational convenience, strength distribution of components at each load application is always adopted in conventional dynamic reliability models, which is determined by the assumed stochastic process. However, the results show that SDPD has considerable influences on fuzzy dynamic reliability of parallel systems. In addition, the failure dependence of components in a system also significantly affects the system fuzzy dynamic reliability.

By defining the SDPD sensitivity function and the failure dependence sensitivity function in this paper, it is found that the fuzzy characteristics of stress have great impacts on the effects of SDPD and failure dependence on fuzzy dynamic reliability. In general, the effects of SDPD and failure dependence of mechanical components in a parallel system on reliability are more sensitive to variation of the right parameter of $c$ than the left parameter of $a$. Moreover, the influences of $a$ and $c$ on SDPD sensitivity and that on 
failure dependence sensitivity are different. The proposed models provide the basis for analytically determining the moment when the peak values of SDPD sensitivity and failure dependence sensitivity appear, which are important to the dynamic reliability evaluation and reliability-based design of mechanical parallel systems. In addition, Monte Carlo simulation is carried out to validate the effectiveness of the proposed models.

\section{Conflict of Interests}

The authors declare that there is no conflict of interests regarding to the publication of this paper.

\section{Acknowledgments}

This work was supported by the Natural Science Foundation of China under Contract no. 51175240, the Natural Science Foundation of China under Contract no. 51175072, and the Natural Science Foundation of China under Contract no. 51335003.

\section{References}

[1] P. D. Herrington, "Stress-strength interference theory for a pinloaded composite joint," Composites Engineering, vol. 5, no. 8, pp. 975-982, 1995.

[2] N. Ranganathan, M. Benguediab, C. Nicolas, G. Henaff, and J. Petit, "Fatigue crack propagation under block loading analysed in terms of equivalent loading concepts," Engineering Fracture Mechanics, vol. 42, no. 1, pp. 59-71, 1992.

[3] L. Xie, J. Zhou, Y. Wang, and X. Wang, "Load-strength order statistics interference models for system reliability evaluation," International Journal of Performability Engineering, vol. 1, no. 1, pp. 23-36, 2005.

[4] J. F. Castet and J. H. Saleh, "Single versus mixture Weibull distributions for nonparametric satellite reliability," Reliability Engineering and System Safety, vol. 95, no. 3, pp. 295-300, 2010.

[5] S. Somasundaram and D. Audsin Mohana Dhas, "Reliability of a dynamic n-unit shared load parallel system under different failure times," Microelectronics Reliability, vol. 37, no. 5, pp. 869871, 1997.

[6] L. A. Zadeh, "Fuzzy sets," Information and Computation, vol. 8, pp. 338-353, 1965.

[7] K. Y. Cai, C. Y. Wen, and M. L. Zhang, "Fuzzy variables as a basis for a theory of fuzzy reliability in the possibility context," Fuzzy Sets and Systems, vol. 42, no. 2, pp. 145-172, 1991.

[8] K. Y. Cai, C. Y. Wen, and M. L. Zhang, "Fuzzy states as a basis for a theory of fuzzy reliability," Microelectronics Reliability, vol. 33, no. 15, pp. 2253-2263, 1993.

[9] K. Y. Cai, C. Y. Wen, and M. L. Zhang, "Posbist reliability behavior of typical systems with two types of failure," Fuzzy Sets and Systems, vol. 43, no. 1, pp. 17-32, 1991.

[10] H.-C. Wu, "Fuzzy reliability analysis based on closed fuzzy numbers," Information Sciences, vol. 103, no. 1-4, pp. 135-159, 1997.

[11] L. V. Utkin, S. V. Gurov, and I. B. Shubinsky, "A method to solve fuzzy reliability optimization problem," Microelectronics Reliability, vol. 35, no. 2, pp. 171-181, 1995.
[12] H.-Z. Huang, M. J. Zuo, and Z.-Q. Sun, "Bayesian reliability analysis for fuzzy lifetime data," Fuzzy Sets and Systems, vol. 157, no. 12, pp. 1674-1686, 2006.

[13] L. Bing, Z. Meilin, and X. Kai, "Practical engineering method for fuzzy reliability analysis of mechanical structures," Reliability Engineering and System Safety, vol. 67, no. 3, pp. 311-315, 2000.

[14] P. Gao, S. Yan, L. Xie, and J. Wu, "Dynamic reliability analysis of mechanical components based on equivalent strength degradation paths," Journal of Mechanical Engineering, vol. 59, no. 6, pp. 387-399, 2013.

[15] Y. Dong, Design of Mechanical Fuzzy Reliability, Machine Industry Press, Beijing, China, 2001.

[16] Y. Dong, "Fuzzy reliability design with random variable and fuzzy variable," Chinese Journal of Mechanical Engineering, vol. 36 , no. 6 , pp. $25-29,2000$.

[17] Q. Jiang and C.-H. Chen, "A numerical algorithm of fuzzy reliability," Reliability Engineering and System Safety, vol. 80, no. 3, pp. 299-307, 2003.

[18] H. T. Nguyen, V. Kreinovich, B. Wu, and G. Xiang, Computing Statistics under Interval and Fuzzy Uncertainty: Applications to Computer Science and Engineering, Springer, 2011. 


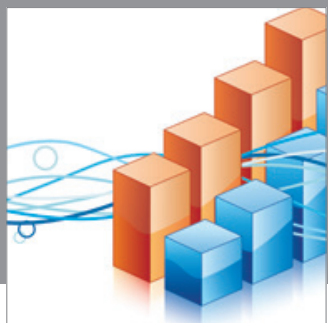

Advances in

Operations Research

mansans

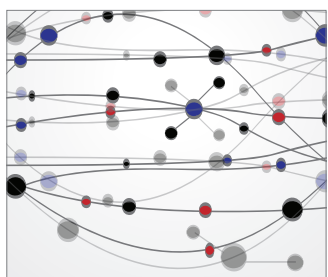

The Scientific World Journal
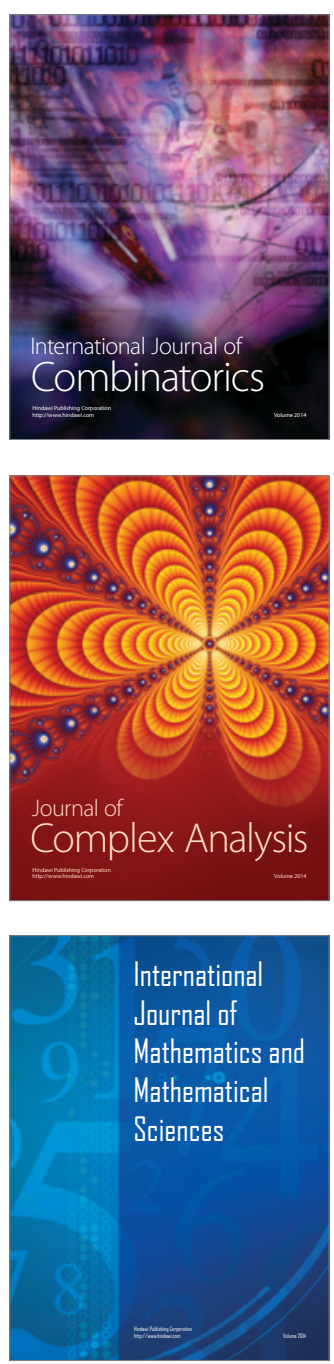
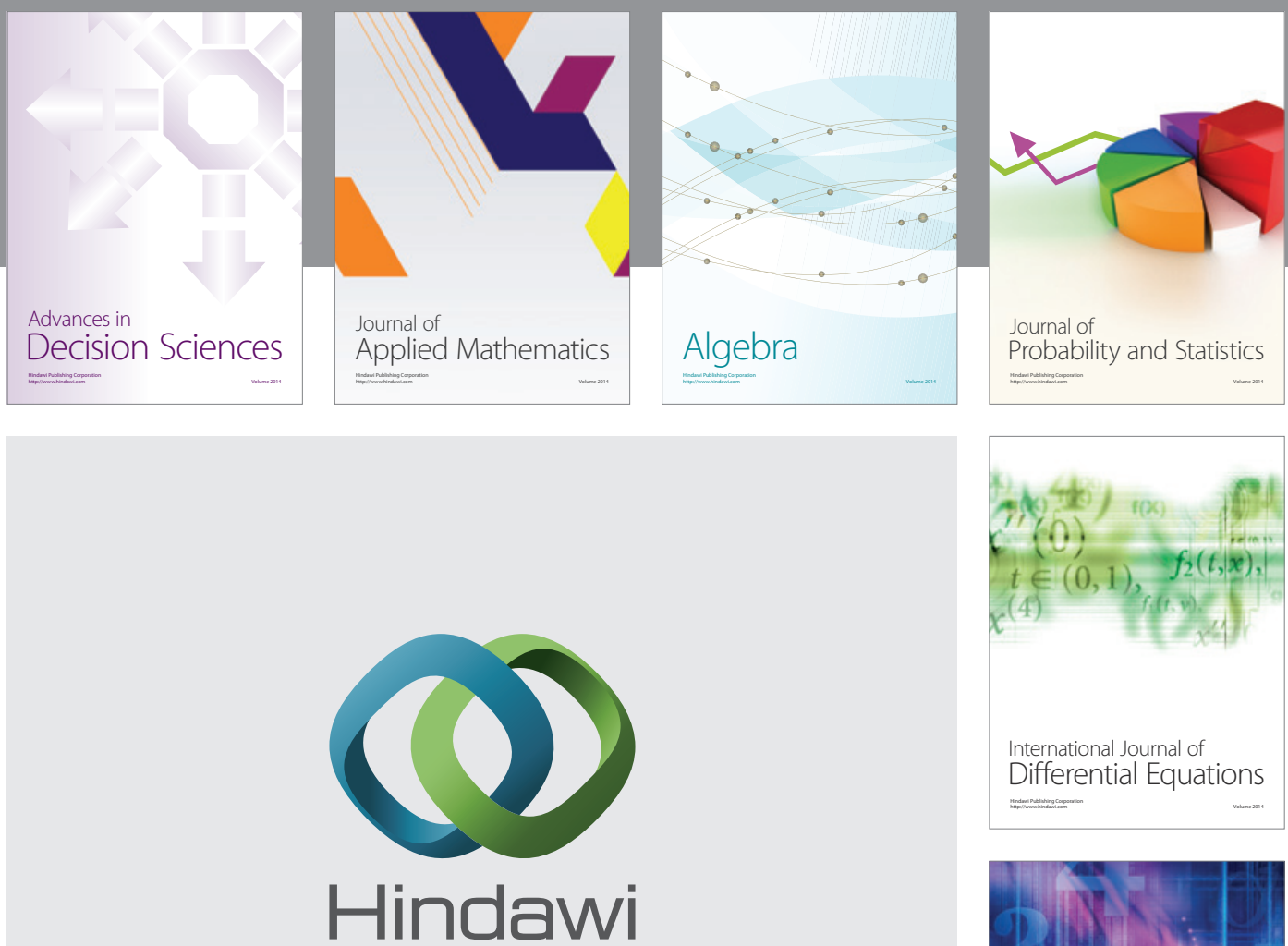

Submit your manuscripts at http://www.hindawi.com
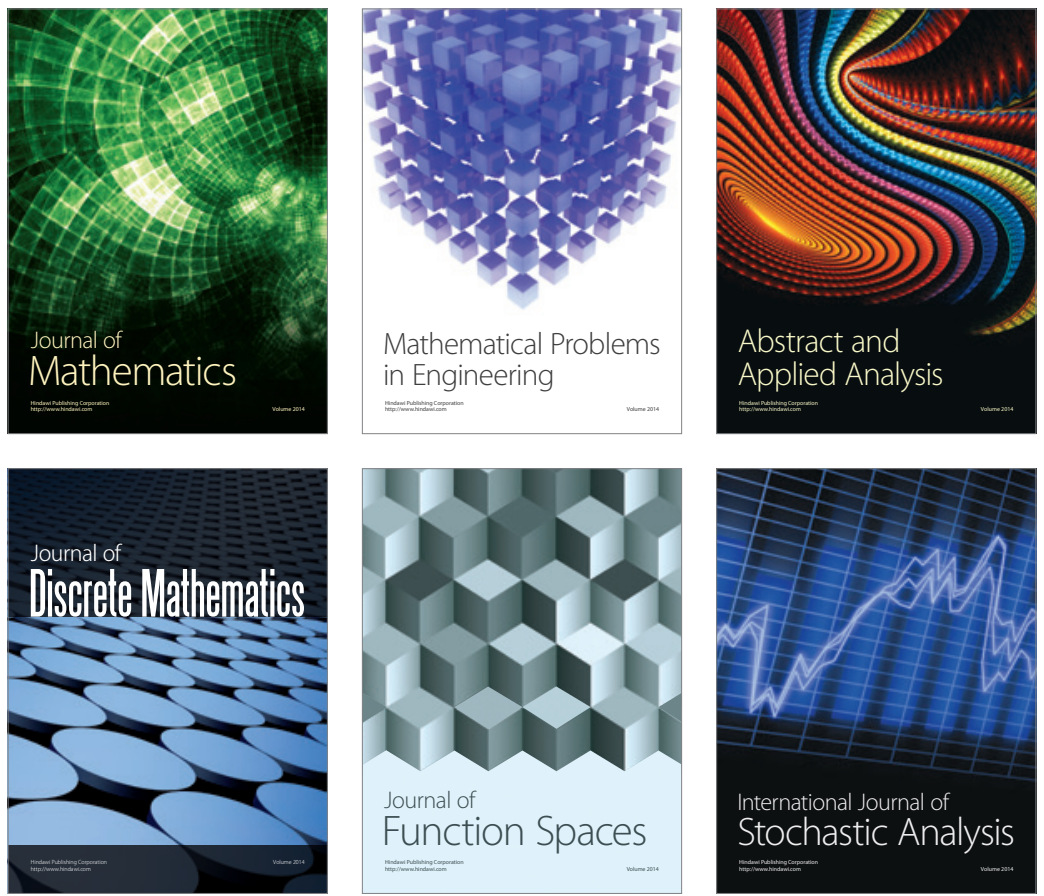

Journal of

Function Spaces

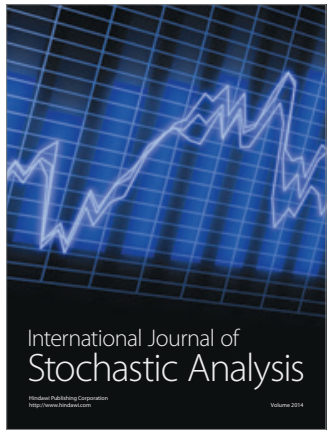

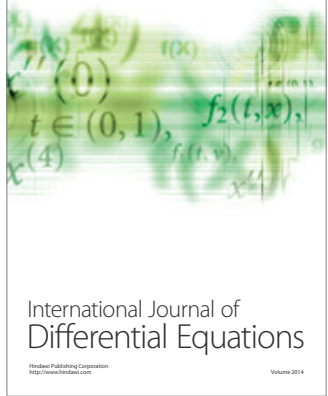
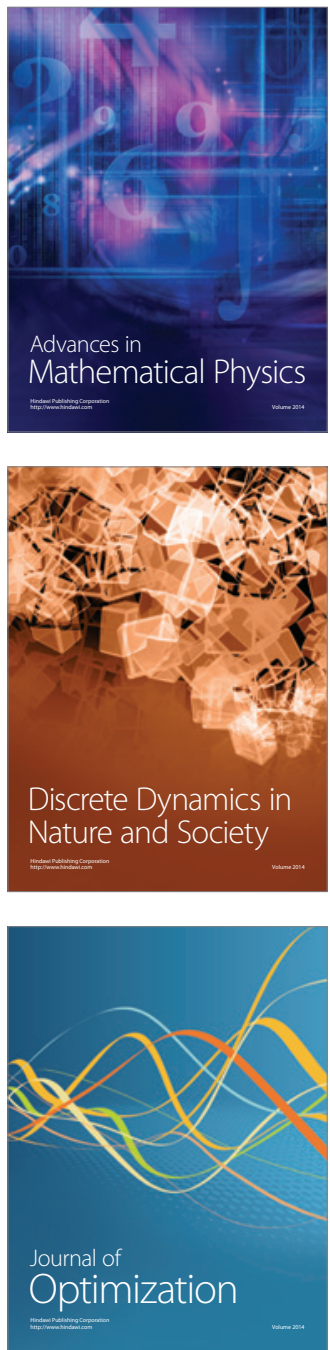